\title{
EFEITOS DO ENXOFRE E DO BORO E DA INOCULAÇÃO EM ALFAFA (Medicago sativa L.)
}

\author{
E. MALAVOLTA \\ T. COURY \\ Livres Docentes e Assistentes de Química Agrícola, \\ E. S. A. "Luiz de Queiroz" - U. S. P. \\ F. GALLI \\ Assistente de Fitopatologia e Microbiologia Agrícola, \\ E. S. A. "Luiz de Queiroz", U. S. P. \\ J. GOMES DA SILVA \\ Seção de Gereais e Leguminosas, Instituto Agronômico do \\ E. S. Paulo, Campinas \\ Tese aprovada no 10.0 Congresso Brasi- \\ leiro de Química, realizado em julho de \\ 1952, no Rio de Janeiro

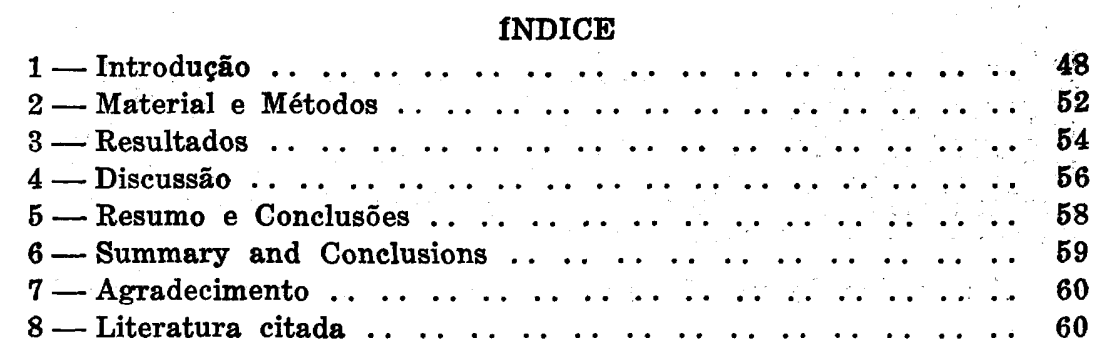




\section{1 - INTRODUÇÃO}

Numerosa é a literatura estrangeira, principalmente americana, sôbre a aplicação do enxofre e do boro em agricultura Daremos a seguir um resumo mencionando apenas os resultados referentes à alfafa os quais sugeriram o ensaio presente. Antes, porém, vamos fazer algumas considerações sôbre o enxofre e o boro na agricultura. 1.1. O enxofre é essencial ao desenvolvimento das plantas que o tem principalmente como proteinas sulfuradas. Estas encerram o S, principalmente, como aminoácido cistina. Tôdas as proteinas vegetais contém enxofre. Como a quantidade dêsse aminoácido nas diferentes proteinas varia consideràvelmente, o mesmo acontece com a proporção de enxofre nas proteinas. Nas proteinas que contém muita cistina, 7 por cento da molécula é constituida de enxofre, enquanto em outras a proporção de $\mathrm{S}$ pode ser menor que $0,01 \%$ (STILES, pág. 282). Em certas plantas, como as crucíferas, o enxofre, em grande proporção se acha no estado de isosulfocianatos alcoólicos (SCNR). Noutras, sob a forma de compostos mais complexos pré-existentes.

A quantidade de $S$ nas plantas é função da espécie considerada e numa mesma planta varia com a parte analisada. MALAVOLTA (1950) estudou a distribuição do enxofre em 35 espécies de plantas cultivadas no Estado de São Paulo, notando de modo geral que a seguinte ordem decrescente para o teor de $\mathrm{S}$ é obedecida :

\section{fôlhas $>$ caule $>$ raízes}

As plantas absorvem enxofre dos solos de preferência como sulfatos. Porém a possibilidade de absorção e aproveitamento de $S$ orgânico foi demonstrada recentemente entre nós (AVERNA SACCA e MALAVOLTA, 1950).

Clarke opina que o enxofre aparece na crosta terrestre na quantidade de $0,06 \%$, em forma elementar ou ligado como sulfatos e sulfetos. Nos solos normais, entretanto, a proporção maior de enxofre se acha em forma orgânica, como foi demonstrado, entre outros, por MALAVOLTA (1950). Em solos do Estado de São Paulo, o teor de sulfatos varia entre $0,0013 \mathrm{~g}$ de $\mathrm{S}$ por $100 \mathrm{~g}$ de solo até um máximo de $0,007 \mathrm{~g}$ de $\mathrm{S}$. As determinações de $\mathrm{S}$ total revelaram um teor mínimo de $0,007 \mathrm{~g}$ de $\mathrm{S} / 100 \mathrm{~g}$ de terra e um máximo de $0,096 \mathrm{~g}$.

As perdas de enxofre nos solos se verificam em consequência das lavagens, isto é, infiltração das águas de gravitação e 
elas podem ser apreciadas com o auxilio de lisímetros. A afamada Estação Experimental de Rothamsted, graças a isso, avalia que o enxofre é arrastado nas águas de drenagem à razão de 20 libras, por acre anualmente. Em Carolina, nos Estados Unidos, reputa-se que a perda semelhante é de 50 libras, por acre e por ano, sendo de 15 a 20 , em Iowa. No Brasil, nada se sabe a êsse respeito.

Note-se que o enxofre é restituido aos solos pela atmosfera. A decomposição de substâncias orgânicas e, principalmente, a queima delas, carvão, turfa, lenha, óleos, lançam no ar enxofre combinado com o oxigênio. As chuvas o devolvem à terra. Nas vizinhanças das grandes cidades, maximé nas industriais, constata-se essa devolução em escala mais acentuada. Em Rothamsted, Inglaterra, o retôrno nessas condições é de 7 libras, por acre, anualmente. Nas proximidades da Universidade de Illinois, 41,5; em Nova Iorque, 41; em Minneapolis, 5 libras apenas.

Sòmente em 1912 é que a América reconheceu o enxofre elementar como fertilizante. $\mathrm{E}$ quando se usa enxofre elementar para êsse fim, supõe-se que êle se transforma nos terrenos, em virtude de microorganismos, assim: $\mathrm{S}+\mathrm{H} 2 \mathrm{O}+\mathrm{O} 2=$ $=\mathrm{H} 2 \mathrm{SO} 3$; $\mathrm{H} 2 \mathrm{SO} 3+\mathrm{CaH} 2(\mathrm{CO} 3) 2=\mathrm{CaSO} 3+2 \mathrm{H} 2 \mathrm{O}+2 \mathrm{CO} 2$; $2 \mathrm{CaSO} 3+\mathrm{O} 2=2 \mathrm{CaSO} 4$. A marcha dessa transformação é admitida por LIPMAN (1911) e a ela dá o nome de sulfatação. O enxofre em pó, é, por via de regra, adicionado de microorganismos oxidantes : é o enxofre inoculado. Para inocular o enxofre elementar usam-se culturas de bactérias do gênero Thiobacillus, do qual a espécie mais bem estudada é Th. thiooxidans, Waksman e Joffe. Th. Thiooxidans oxida ràpidamente o enxofre elementar a H2SO4 sem formação de produtos de grau de oxidação intermediário (WAKSMAN, 1927, pág. 607) :

$$
2 \mathrm{~S}+3 \mathrm{O} 2+2 \mathrm{H} 2 \mathrm{O}=2 \mathrm{H} 2 \mathrm{SO} 4+282 \mathrm{Cal}
$$

Para o caso particular da alfafa mencionem-se os seguintes ensaios :

ADAMS (1924) estudando a influência do enxofre empregado na proporção de 0 a 3.000 lbs. por acre sôbre a germinação e o crescimento inicial de alfafa notou : pequeno efeito na germinação e no crescimento inicial exceto para as grandes aplicações, casos em que houve diminuição no crescimento.

BROWN (1917) no estado de Oregon comparou os efeitos do gesso e do enxofre elementar com os seguintes resultados: uma aplicação de $100 \mathrm{lbs}$. de gesso produziu $7.522 \mathrm{lbs}$. de feno 
por acre, ao passo que nos canteiros não tratados obtiveram-se 2.104 lbs. Uma aplicação de $16,6 \mathrm{lbs}$. de $\mathrm{S}$ elementar - quantidade equivalente ao teor de $\mathrm{S}$ nas $100 \mathrm{lbs}$. de gesso - produziu $7.650 \mathrm{lbs}$. de feno por acre, havendo um excesso de $890 \mathrm{lbs}$. sôbre a testemunha.

BRUCE (1925) não notou nenhum aumento acentuado na colheita nem no desenvolvimento radicular de alfafa como resposta à adubação com enxofre o que indica que o elemento não é o fator limitante à produção dessa leguminosa no tipo de solo usado.

ERDMAN (1923) notou influência benéfica da aplicação de $200 \mathrm{lbs}$. por acre na produção de feno de alfafa.

HADDOCK e VANDECAVEYE (1945) verificaram ser o enxofre o elemento limitante para a produção de alfafa nos tipos de solo estudados (Olympic loam e Lynden fine sandy loam).

No Estado de Iowa (ANÔNIMO, 1925) foi notada, em 6 tipos de solos, influência considerável sôbre a produção de alfafa.

JOFFE e MC LEAN (1922) relataram ensaios com enxofre feitos no Estado de Oregon nos quais houve aumento de produção como consequência de resposta favorável dos solos.

Ensaios executados na Estação Experimental de Montana, U. S. A., mostraram que o S produziu um aumento de 1,92 tons/acre, ao passo que o gesso, 1,35 tons/acre (ANÔNIMO, 1925); foi acentuado também o aumento na quantidade de proteina.

NEIDIG e colaboradores (1923) verificaram que o $\mathrm{S}$ aumentou claramente a colheita de alfafa nos solos da parte não irrigada do Estado de Idaho, U.S.A., mas não teve efeito marcado nos solos áridos.

Aplicando enxofre na proporção de $75 \mathrm{lbs} / \mathrm{acre}$, NELLER (1925) notou influência benéfica do enxofre em pó sôbre a produção de alfafa, sendo que o efeito foi mais acentuado quando o material era distribuido a lanço e não mexido com a terra antes do plantio.

Da investigação de OLSON e ST. JOHN (1921) sôbre o enxofre como alimento da planta resultou a conclusão de que a alfafa utiliza mais enxofre do que fósforo.

Uma série de trabalhos conduzidos no Estado de Oregon, U.S.A., (ANONIMO, 1919-20, 1921-22) mostraram que : o enxofre foi usado no período 1918-20 numa área de 16.000 acres dando um aumento médio de 1 ton/acre; aproximadamente 100.000 acres do mesmo Estado podem dar 1 ton adicional na colheita anual de alfafa desde que se apliquem $100 \mathrm{lbs}$. de S cada quatro anos. 
A resposta acentuada da alfafa ao $\mathrm{S}$ nos solos de Oregon foi objeto de vários relatórios por POWERS $(1923,1927,1930)$.

Colheitas consideràvelmente aumentadas graças à adubação com $S$ foram mencionadas por REIMER (1914); REIMER e TARTAR (1919) que relataram aumentos de 50 a 1.000 por cento em vários solos de Oregon recomendando aplicações de 40 a $50 \mathrm{lbs}$. de $\mathrm{S}$ por acre juntamente com $200 \mathrm{lbs}$. de fosfato de rocha como altamente proveitosas.

ST. JOHN (1923) verificou, em condições de casa de vegetação, que o aumento inicial na produção determinado pela aplicação de enxofre só se mantinha quando novas aplicações eram feitas.

Pequenos acréscimos nas colheitas foram observados por SHEDD (1917) no Estado de Kentucky, U.S.A.

Nenhum efeito da adubação sulfurada foi notado por TOEVS e BAKER (1939) no Estado de Idaho, U.S.A.

Trabalhos na Washington Exp. Sta. (ANÔNIMO, 1919) relataram um aumento de $200 \%$ na produção.

1.2. Só depois da 1a. Guerra Mundial é que se estabeleceu o caráter essencial do boro para as plantas (WARINGTON, 1923; SOMMER and LIPMAN, 1926; e outros). Na ausência de $\mathrm{B}$ o desenvolvimento do tecido meristemático e a nodulação das leguminosas não ocorrem devidamente. SCRIPTURE e MC HARGUE (1945) concluiram que o B é funcional no metabolismo e translocação dos carbohidratos e HOAGLAND (1944) sugere que êle tem importância na formação de compostos pécticos e entra em combinação com açúcar e ácidos orgânicos através de grupos OH adjacentes. DENNIS (1937) apresentou evidência mostrando que as doenças "browning" da couve-flor, "brown heart" do nabo, "roan" da beterraba, "internal cork" das maçãs, "cracked stem" do aipo e "yellows" da alfafa, são causadas por falta de boro.

Bórax (tetraborato de sódio) é o produto contendo boro usado mais comumente. Em trabalhos experimentais, entretanto, também o ácido bórico, borosilicatos finamente pulverizados e o borato de potássio se mostram satisfatórios. Para a maioria das culturas, pode ser empregado na dose de 10 a 50 lhs. de bórax/acre, misturado a outros adubos. Para árvores frutíferas o boro pode ser aplicado em pulverizações juntamente com outras substâncias usadas no combate a pragas e moléstias. Para qualquer cultura, porém, as aplicações podem ser mais pesadas quando os solos possuem muita matéria orgânica, alta capacidade de troca ou elevado $\mathrm{pH}$. Aplicações de borax nas proporções de $10 \mathrm{lbs}$. para solos leves e $20 \mathrm{lbs}$. para solos pe- 
sados são recomendadas por AHLGREN et al. (1945) para corrigir deficiências de boro no Estado de New Jersey, U.S.A.

BAUR et al. (1941), entretanto, aconselham o emprêgo de quantidades bem maiores - 30 a 60 lbs. de borax - com o mesmo fim para os solos de Washington.

BROWN e KING (1939) usaram 20 lbs. de borax/acre para controlar carência de $\mathrm{B}$ obtendo além disso um aumento de $16 \%$ na produção.

DUNKLEE e MIDGLEY (1943) escreveram que só em 2 por cento dos 850.000 acres de terra usada para feno em Vermont possuem quantidade suficiente de B para alfafa recomendando por isso o emprêgo de $40 \mathrm{lbs} /$ acre cada 3 ou 4 anos.

Usando $15 \mathrm{lbs}$. de borax/acre, GRIZZARD e MATTHEWS (1942) obtiveram aumento acentuado na colheita de feno e boa quantidade de semente ao passo que as plantas que não receberam borax deixaram de produzir sementes.

Ensaio de HUTCHESON e COCKE (1941) mostraram grandes acréscimos nas colheitas mediante aplicação de 10-20 lbs. de borax/acre em solos deficientes de $B$ na Virginia podendo o fertilisante ser usado após a semeadura ou mesmo depois do primeiro corte.

MC LARTY et al. (1937) trabalhando na British Columbia verificaram que a alfafa deficiente em boro cultivada em condições de campo possuia 6,9 p.p.m. de boro ao passo que as plantas normais tinham 29,4 p.p.m.

A importância do B para a produção de sementes em alfafa foi constatada também por PILAND et al. (1944).

WASHKO (1943) verificou que a aplicação combinada de $\mathrm{K}$ e borax era mais efetiva para combater carência de $\mathrm{B}$ (alfafa yellows) em solos do Tennesse, aumentando consideràvelmente a colheita de feno, altura da planta e a longevidade.

1.3. Não encontramos na literatura brasileira à nossa disposição nenhuma referência ao uso do enxofre e do boro na adubação de alfafa. As determinações de MALAVOLTA (1950), por outro lado, haviam revelado ser os solos do Estado de São Paulo mais pobres em enxofre que a média dos solos americanos; este fato e mais os resultados surpreendentes de REIMER e TARTAR (1919) nos levaram a instalar o presente ensaio.

\section{2 - MATERIAL E MÉTODOS}

2.1. Ensaio de campo. O ensaio foi feito numa pequena área de terra roxa misturada localizada no aviário da E. S. A. "Luiz de Queiroz", U.S.P. Agradecemos ao Prof. A. Paravicini Torres a permissão para usar esse terreno. 
O delineamento escolhido foi um quadrado latino de $6 \times 6$ com os seguintes tratamentos:

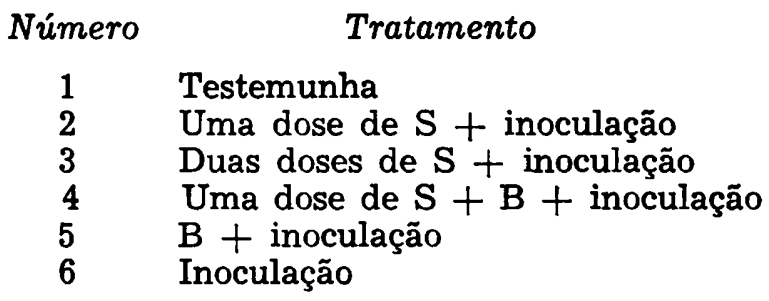

Os canteiros mediam $5 \times 5 \mathrm{~m}$. Antes da instalação do ensaio foram distribuidas uniformente sôbre tôda a área de $1800 \mathrm{~m} 2$ aproximadamente, os seguintes materiais

1 tonelada de calcáreo $120 \mathrm{~kg}$ de farinha de óssos (28-30\% P2O5 $60 \mathrm{~kg}$ de serranafosfato (28\% P2O5 total) $60 \mathrm{~kg}$ de cloreto de potássio $(60 \% \mathrm{~K} 2 \mathrm{O})$ 4,5 toneladas de esterco de célula Beccari.

O enxofre elementar foi aplicado na proporção de 1 tonelada por hectare para a dose simples e 2 toneladas para a dupla tendo sido usado o enxofre molhável; o borax foi empregado na proporção de $15 \mathrm{~kg} / \mathrm{ha}$. Esses dois materiais foram distribuidos cuidadosamente sôbre os canteiros e a seguir misturados com a terra por meio de um rastêlo.

A inoculaçã̀o foi feita nas sementes de alfafa usando-se as suspensões de lavagem de 7 tubos de ensaio com 7 diferentes linhagens de Rhizobium meliloti; a quantidade total de liquido usado era aproximadamente, $150 \mathrm{cc}$.

Em cada canteiro foram feitos 24 sulcos de $5 \mathrm{~cm}$ de profundidade e espaçados de $20 \mathrm{~cm}$; cada sulco recebeu $2,29 \mathrm{~g}$ de sementes tendo a semeadura sido feita dez dias após a aplicação de $\mathrm{S}$ e $\mathrm{B}$.

2.2. Ensaio em vasos. Colheu-se terra do mesmo lugar em que foi feito o ensaio de campo para utilizá-la no experimento em vasos. A coleta se deu após a distribuição da adubação básica em cobertura. Foram usados 20 vasos de Mitscherlich, de tamanho grande, recebendo cada um $12 \mathrm{~kg}$ de solo e tratamento como segue : 
... N.

Tratamento

1 - 2 Testemunha

3 - 4 Inoculação

5 a 8 Uma dose de $\mathrm{S}+$ inoculação

9 a 12 Duas doses de $S+$ inoculação

13 a 16 Uma dose de $S+B+$ inoculação

17 a $20 \quad \mathrm{~B}+$ inoculação

O enxofre e o boro foram aplicados em doses equivalentes às usadas no ensaio de campo, considerando-se para isso a área do vaso. Praticada a adubação com $\mathrm{S}$ e $\mathrm{B}$, manteve-se durante 15 dias a humidade a $50 \%$ do poder de embebição e a seguir foi feita a semeadura de $1 \mathrm{~g}$ de sementes por vaso. Nos vasos que receberam enxofre se fez inoculação de bactérias oxidantes do $\mathbf{S}$ por meio duma suspensão de terra fresca inoculada prèviamente com $\mathrm{S}$ elementar.

\section{3 - RESULTADOS}

3.1. Ensaio de campo. Este ensaio foi instalado em 10-6-50. Foram feitos quatro cortes obtendo-se para cada tratamento (soma das 5 repetiçōes) as produções abaixo:

\section{QUADRO I}

\begin{tabular}{c|c|c|c|c|c}
\hline \multirow{2}{*}{ Tratamontos } & \multicolumn{4}{|c|}{ CORTE } & \multirow{2}{*}{ Total } \\
\cline { 2 - 4 } & $26-11-50$ & $23-1-51$ & $9-3-51$ & $9-5-51$ & \\
\hline & $182,6 \mathrm{~kg}$ & $183,2 \mathrm{~kg}$ & $153,4 \mathrm{~kg}$ & $65,6 \mathrm{~kg}$ & $584,8 \mathrm{~kg}$ \\
$\mathbf{1}$ & $190,0 \mathrm{~kg}$ & $211,0 \mathrm{~kg}$ & $194,0 \mathrm{~kg}$ & $78,8 \mathrm{~kg}$ & $674,6 \mathrm{~kg}$ \\
$\mathbf{2}$ & $211,8 \mathrm{~kg}$ & $233,4 \mathrm{~kg}$ & $206,8 \mathrm{~kg}$ & $96,3 \mathrm{~kg}$ & $717,3 \mathrm{~kg}$ \\
$\mathbf{5}$ & $193,2 \mathrm{~kg}$ & $192,4 \mathrm{~kg}$ & $192,4 \mathrm{~kg}$ & $76,4 \mathrm{~kg}$ & $641,2 \mathrm{~kg}$ \\
$\mathbf{4}$ & $191,8 \mathrm{~kg}$ & $208,2 \mathrm{~kg}$ & $183,8 \mathrm{~kg}$ & $73,2 \mathrm{~kg}$ & $656,0 \mathrm{~kg}$ \\
$\mathbf{5}$ & $164,8 \mathrm{~kg}$ & $168,4 \mathrm{~kg}$ & $145,8 \mathrm{~kg}$ & $65,2 \mathrm{~kg}$ & $534,2 \mathrm{~kg}$ \\
$\mathbf{6}$ & &
\end{tabular}

Atribuimos o notável abaixamento na produção verificado no quarto corte à sêca reinante no período.

A análise estatística foi feita apenas com os dados referentes às colheitas totais por canteiro que se acham no quadro seguinte (os algarismos superiores indicam os tratamentos) : 


\section{QUADRO II}

\begin{tabular}{c|c|c|c|c|c}
\hline $\mathbf{A}$ & $\mathbf{B}$ & $\mathbf{C}$ & \multicolumn{1}{|c|}{$\mathbf{D}$} & $\mathbf{E}$ & $\mathbf{F}$ \\
\hline 1 & 2 & 3 & 4 & $\mathbf{5}$ & $\mathbf{6}$ \\
96,6 & 123,6 & 120,0 & 103,2 & 94,0 & 86,0 \\
\cline { 1 - 5 } 2 & 5 & $\frac{4}{6}$ & $\mathbf{6}$ & $\mathbf{3}$ & $\mathbf{1}$ \\
80,8 & 114,0 & 105,8 & 57,4 & 100,3 & 86,8 \\
\hline 3 & 1 & 6 & 5 & 4 & $\mathbf{2}$ \\
117,0 & 95,4 & 90,4 & 84,6 & 88,0 & 87,4 \\
\hline 4 & 6 & 1 & 3 & 2 & 5 \\
102,2 & 95,8 & 114,2 & 130,6 & 119,8 & 108,8 \\
\hline 5 & 4 & 2 & 1 & 6 & 3 \\
$1.28,8$ & 143,4 & 145,6 & 97,8 & 82,4 & 125,0 \\
\hline 6 & 3 & 5 & 2 & 1 & 4 \\
122,2 & 124,4 & 125,8 & 117,4 & 94,0 & 98,6 \\
\hline
\end{tabular}

A análise da variância está resumida no Quadro III :

\section{QUADRO III}

\begin{tabular}{l|r|r|r|r|l}
\hline $\begin{array}{c}\text { Fonte de } \\
\text { variação }\end{array}$ & nf & $\begin{array}{c}\text { Soma dos } \\
\text { quadrados }\end{array}$ & Variância & $\mathbf{F}$ & Significân. \\
\hline & & & & & \\
Linhas & 5 & $4.430,85$ & 886,17 & & \\
Colunas & 5 & $2.863,59$ & 572,71 & & \\
Tratamen. & 5 & $3.891,45$ & 778,29 & 7,361 & signif. - 1\% \\
Resíduo & 20 & $2.114,79$ & 105,73 & & \\
\hline
\end{tabular}

Damos a seguir as produções totais por tratamento em quilogramas e em percentagem

QUADRO IV

\begin{tabular}{c|c|c}
\hline \multirow{2}{*}{ Tratamento } & \multicolumn{2}{|c}{ PRODUÇÃO } \\
\cline { 2 - 3 } & Quilos & $\%$ \\
\hline & & $\%$ \\
1 & 584,8 & 100 \\
2 & 674,6 & 114 \\
3 & 641,2 & 122 \\
4 & 717,3 & 109 \\
5 & 656,0 & 111 \\
6 & 534.9 & 90 \\
\hline
\end{tabular}


A comparação entre as médias feita pelo processo gráfico descrito por RODRIGUES DE CARVALHO (1946, pp. 84-85) (ver Fig. 1) mostrou que : o tratamento 3 (duas doses de $\mathrm{S}+$ inoculação) difere significativamente de todos os demais; não há diferença significativa entre os tratamentos 2,4 e 5 uma vez, que os segmentos - representativos se superpõem; os tratamentos 1 e 6 , respectivamente testemunha e inoculação, diferem, entre sí, e, também, dos demais tratamentos.

3.2. Ensaio em vasos instalado em 25-7-50. No quadro seguinte estão os resultados de cada tratamento (produção média por tratamento) :

QUADRO V

\begin{tabular}{|c|c|c|c|c|c|}
\hline \multirow{2}{*}{ Tratamentos } & \multicolumn{4}{|c|}{ Corte - gramas } & \multirow{2}{*}{$\begin{array}{l}\text { gramas } \\
\text { Média }\end{array}$} \\
\hline & 4-12-50 & $16-1-51$ & $1-3-51$ & $30-4-51$ & \\
\hline Testem. & 82,5 & 151,0 & 65,5 & 56,0 & 89,0 \\
\hline lação & 115,5 & 141,7 & 69,7 & 43,0 & 93,1 \\
\hline $\mathrm{s}+\mathrm{B}+$ & 71,7 & 92,2 & 48,5 & 35,5 & 62,0 \\
\hline $\begin{array}{c}\text { inocula- } \\
\text { ção } \\
\mathrm{B}+\text { inocu- }\end{array}$ & 92,7 & 127,0 & 68,2 & 48,0 & 61,3 \\
\hline lação & 93,2 & 119,2 & 65,0 & 42,7 & 73,5 \\
\hline
\end{tabular}

\section{4 - DISCUSSAO}

4.1. Ensaio de campo. Embora a análise estatística tivesse revelado grande heterogeneidade do terreno, houve diferença significativa entre os tratamentos que se classificaram na seguinte ordem decrescente :

1.o - duas doses de $S+$ inoculação; 2.0 - uma dose de $S$ + inoculação, uma dose de S + B inoculação, B + inoculação; 3.o - testemunha; 4.0 - inoculação.

Entretanto, do ponto de vista econômico, a situação fica alterada, como se verá no Quadro VI. Os cálculos foram feitos considerando-se os preços dos adubos, da semente e da mão de obra (preparo de solo, adubação, semeadura, capinas e quatro 
cortes) por ocasião do ensaio; para determinar o valor do feno considerou-se um rendimento de $30 \%$ e o preço de $\operatorname{Cr} \$ 2,00$ por quilo.

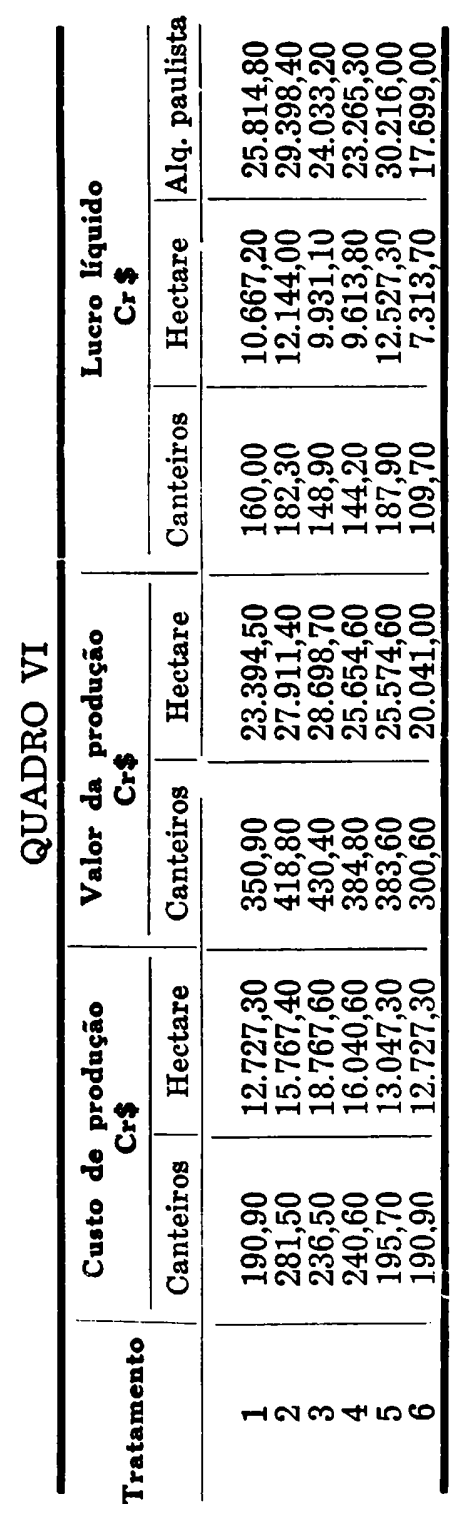


O quadro VI mostra que o tratamento mais conveniente do ponto de vista econômico é o tratamento n. 5 , ou seja, uma dose de $\mathrm{B}+$ inoculação. $\mathrm{O}$ fato de o tratamento n. 3 - duas doses de $\mathrm{S}+$ inoculação ter garantido as colheitas mais altas sugere que o terreno estudado e talvez as terras do mesmo tipo espalhadas pelo Estado não apresentem a totalidade da reserva de $\mathbf{S}$ assimilável exigida pela alfafa. Por outro lado, a resposta da alfafa ao tratamento com boro, ainda que não fosse tão acentuada quanto à anterior, - no que se refere à produção, foi, também, significativa.

Julgamos, para o fato do tratamento inoculação, ter dado resultados inferiores à testemunha, a seguinte explicação: a matéria orgânica adicionada com esterco de célula Beccari, através da nitrificação, deve ter fornecido uma reserva de nitrato a qual não permitiria a fixação de $\mathrm{N}$ atmosférico pelas bactérias o que, aliás, foi verificado através da fraca nodulação encontrada nas amostras de planta coletadas para observação. Esta hipótese, evidentemente, exige uma expíicação complementar, que é a seguinte: uma quantidade abundante de nitrato no solo determina um teor alto do mesmo radical na planta; aqui se dá a redução do NO3- e a formação de aminoácidos, sendo, portanto, os carbohidratos presentes consumidos ràpidamente, nada ou pouco sobrando para as bactérias. Como consequência, a fixação do $\mathrm{N}$ não se dá e a simbiose é transformada em parasitismo, com prejuizo para o hospedeiro.

4.2. Nos vasos verificou-se a superioridade da dose simples de $\mathrm{S}$, combinada com inoculação sôbre a dose dupla + inoculação. Atribuimos esse fato ao abaixamento exagerado no $\mathrm{pH}$ como seria lícito esperar dadas as condições ideais para a atividade das bactérias oxidantes do $\mathrm{S}$ a $\mathrm{H} 2 \mathrm{SO} 4$ garantidas nos vasos : a acidez excessiva prejudicaria a alfafa que prefere $\mathrm{pH}$ pouco abaixo de 7 .

\section{5 - RESUMO E CONCLUSOES}

Os autores relatam, neste trabalho, os resultados de um ensaio de campo e em vasos realizado para verificar a influência da aplicação de $\mathrm{S}$ e $\mathrm{B}$ e da inoculação na produção de alfafa em terra roxa misturada. No ensaio, além da adubação fundamental PKCa e matéria orgânica, empregou-se $\mathbf{S}$ nas proporções de 1.000 e $2.000 \mathrm{~kg} /$ ha e borax na quantidade de $15 \mathrm{~kg} / \mathrm{ha}$; o delineamento escolhido no campo foi um quadrado latino de $6 \times 6$ com os seguintes tratamentos: 


\begin{tabular}{cl} 
Número & \multicolumn{1}{c}{ Tratamento } \\
1 & Testemunha \\
2 & Uma dose de S + inoculação \\
3 & Duas dose de S + inoculação \\
4 & Uma dose de S + B + inoculação \\
5 & B + inoculação \\
6 & incculação.
\end{tabular}

Foram feitos 4 cortes, tendo o ensaio durado 11 meses. O experimento em vasos, em linhas gerais, confirma os resultados de campo. As seguintes conclusões podem ser tiradas: crescente :

5.1. A classificação dos tratamentos foi, em ordem de-

1.0 - duas doses de $S+$ inoculação; 2.0 - uma dose de $\mathrm{S}+$ inoculação, $\mathrm{S}+\mathrm{B}+$ inoculação e $\mathrm{B}+$ inoculação (esses três tratamentos não diferiram significativamente); 3.0 - testemunha; 4.0 - inoculação.

5.2 A produção obtida com o tratamento $2 \mathrm{~S}+$ inoculação foi de 22 por cento maior que a testemunha, o que sugere que a reserva de $\mathrm{S}$, no solo estudado, não é suficiente para garantir colheitas máximas de alfafa.

5.3 Do ponto de vista econômico, o melhor tratamento foi B + inoculação, o qual possibilita um lucro líquido de .. . . . . Cr\$30.216,00 por alqueire por ano.

5.4 Com base nos resultados apresentados recomendamos, em solos do mesmo tipo, a seguinte adubação para alfafa :

5 tons. de .calcáreo/hectare

$300 \mathrm{~kg}$ de serranafosfato e $600 \mathrm{~kg}$ de hiperfosfato/hectare

$300 \mathrm{~kg}$ de cloreto de potássio/hectare

$15 \mathrm{~kg}$ de borax/hectare

e, ainda, uma adubação orgânica média, se o solo for muito pobre em materia orgânica. Devido ao preço e à aquisição dificil da farinha de ossos, propuzemos a sua substituição pelo hiperfosfato, adubo que se tem comportado satisfatoriamente entre nós.

\section{6 - SUMMARY AND CONCLUSIONS}

This paper deals with the results of a pot and plot experiment which was carried out to determine the influence of sulphur and boron and the effect seed inoculation with Rhizobium meliloti in the yield of alfafa. Sulphur was applied as flower of sulphur at the rates of 1,000 and $2,000 \mathrm{~kg}$ por hectare; boron was employed in the proportion of $15 \mathrm{~kg}$ of borax per 
hectare; both sulphur and boron were distributed broadcast before planting; the experimental design chosen for the field trial was a latin square of $6 \times 6$ with the following treatments :

Num
1
2
3
4
5
6

\section{Treatment}

Control

One dosis $\mathbf{S}+$ inoculation

Two dosis $\mathrm{S}+$ inoculation

One dosis S + B + inoculation

$\mathrm{B}+$ inoculation

inoculation

The crop supplied four cuttings in an eleven months period. The pot experiment nearly confirmed the plot one. The following conclusions can be drawn:

6.1. The classification of treatments in a decrescent order was : 1.0 - two doses $\mathrm{S}+$ inoculation; 2.0 - one dosis $\mathrm{S}+$ inoculation, $\mathrm{S}+\mathrm{B}+$ inoculation, and $\mathrm{B}+$ inoculation (these treatmente were not statistically different); 3.0 - control; 4.0 - inoculation;

6.2 The vield due to the treatment two dosis $\mathrm{S}+$ inoculation was 22 per cent higher than the control one, a fact that suggests that the S supply in the soil studied ("terra roxa misturada") is not sufficient for the total requirements of alfafa;

6.3. From an economical point of view the best treatment was: one dosis B + inoculation which permits a net gain of Cr\$ 12.527,30 per hectare per year;

6.4. Based on the mentioned results we recommend in soils of same type the following fertilization for alfafa.

5 tons limestone/hectare

$300 \mathrm{~kg}$ serranafosfato and $600 \mathrm{~kg}$ hiperfosfato/ha

$300 \mathrm{~kg}$ muriate of potash $/ \mathrm{ha}$

$15 \mathrm{~kg}$ borax/ha

and a medium organic manuring if the soil is very poor in organic matter.

\section{8 - LITERATURA CITADA}

ADAMS, H. R. 1924 - Some effects of sulphur on crops and soils. Soil Sci. 18:111.

AHLGREN, G. H., H. B. Sprague and F. E. Bear. 1945 - Growing alfalfa in New Jersey. New Jersey Sta. Bul. 718.

ANONIMO. 1919-20 - Oregon soil investigations 1918-1920. Oregon Sta., Soil Insvest. Bien. Rpt. 1919-20.

ANONIMO. 1921-22. - Soil fertility investigations (at the Oregon Station). Oregon Sta. Bien. Rept. 1921-22. 
ANONIMO. 1925 - Soil studies at the Iowa Station. Iowa Sta. Rpt. 1925 : 16.

ANONIIMO. 1925 - Effect of sulphur on alfalfa and clover. Montana Sta. Rpt. 1925: 24.

AVERNA-SACCA, Rosário e E. Malavolta. 1950 - Absorção de enxofre orgânico pelo tomateiro. Esc. Sup. Agr. "Luiz de Queiroz" (Piracicaba) Bol. n. 6.

BAUR, Karl, Glenn A. Huber and L. C. Wheeting. 1941 - Boron deficiency of alfalfa in western Washington. Wash. Agr. Expt. Sta. Bul. 39: 5.

BROWN, G. G. 1917 - Alfalfa fertilizers. Oregon Sta. Bul. 141: 55.

BROWN, B. A. and Allan King. 1939 - Soil conditions under which alfalfa responded to boron. Soil Sci. Soc. Am. Proc. $4: 310$.

BRUCE, O. C. 1925 - The relation of sulphur to alfalfa production. Jour. Agr. Res. $30: 937$.

DENNIS, R. W. G. 1937 - A review of developments in the applications of boron in agriculture and horticulture. Fertil., feedingst. and farm. supp. J., Sept. 8, 12, Oct. 6,20.

DUNKLEE, D. E. and A. R. Midgley. $1943-$ Need and use of boron for alfalfa. Vermont Sta. Bul. 501.

ERDMAN, L. W. 1923 - The effect of gypsum on Iowa soils. Soil Sci. $15: 137$.

GRIZZARD, A. L. and E. M. Matthews. 1947 - The effect of boron on seed production of alfalfa. J. Am. Soc. Agron. $34: 365$.

HADDOCK, J. L. and S. C. Vandecaveye. 1946 - Yield and chenical composition of alfalfa on two western Washington soil types. Soil Sci. Soc. Am. Proc. $10: 129$.

HOAGLAND, D. R. 1944 - Lectures on the inorganic nutrition of plants. Chronica Botanica, Walthan, Mass.

HUTCHESON, T. B. and R. P. Cocke. 1941 - Effects of boron on yield and duration of alfalfa. Virginia Polytech. Insti. Blacksburg) Agr. Expt. Sta. Bul. 336 : 1.

LIPMAN, J. G. 1911 - Suggestions concerning the terminology of soil bacteria. Bot. Gaz. $51: 454$.

MALAVOLTA, Eurípedes. 1950 - Estudos sôbre o enxofre Tese. (Piracicaba).

MC LARTY, H. R., J. G. Wilcox and C. G. Woodbridge. $1937-$ Yellowing of alfalfa due to boron deficiency. Sci. Agr. 17 : 515.

NEIDIG, R. E., G. R. Mc Dole and H. P. Magnuson. 1923 - Effect of sulphur, calcium, and phosphorus on the yield and composition of alfalfa on six types of Idaho soils. Soil Sci. $16: 127$. 
NELLER, J. R. 1925 - The influence of sulphur and gypsum upon the composition and yield of legumes. Washington Col. Sta. Bul. 190 : 5.

OLSON, G. A. and J. L. St. John. 1921 - An investigation of sulphur as a plant food. Washington Sta. Bul. 165.

PILLAND, J. R., C. F. Ireland and H. M. Reisenauer. $1944-$ The importance of borax in legume seed production in the south. Soil Sci. 57 : 75.

POWERS, W. L. 1923 - Sulphur in relation to soil fertility. Oregon Sta. Bul. 199 : 5.

POWERS, W. L. 1927 - Studies of sulphur in relation to the soil solution. Calif Univ. Pubs. Agr. Sci. 5: 119.

POWERS, W. L. 1930 - The role of suphur in plant nutrition. J. Am. Soc. Agron. April 1930. (Abs. in Oregon Sta. Biem Rpt. 1929-30: 46).

REIMER, F. C. 1914 - Sulphur as fertilizer for alfalfa. Pacific Rural Press 87: 717.

REIMER, F. C. and H. V. Tartar. 1919 - Sulphur as a fertilizer for alfalfa in Southern Oregon. Oregon Sta. Bul. 165: 5.

RODRIGUES de Carvalho, M. J. 1946 - A estatística na experimentação agrícola. Livraria Sá da Costa, Lisbôa.

SCRIPTURE, P. N. and G. S. Mc Hargue. 1945 - Boron supply in relation to carbohydrate metabolism and distribution in the radish. J. Am. Soc. Agron. 37: 360.

SHEDD, O. M. 1917 - Effect of sulphur on different orops and soils. U. S. Dept. Agr. Jour. Agr. Res. 11 : 91.

SOMMER, A. L. and C. B. Lipman. 1926 - Evidence on the indispensable nature of zinc and boron for higher green plants. Plant Physiol. 1 : 231.

ST. JOHN, J. L. 1923 - Sulphur as a plant food. Washington Col. Sta. Bul. 180 : 18.

STILES, Walter. 1936 - An introduction to the principles of plant physiology. Methen and Co. Ltd. London.

TOEVS, John L. and G. Orien Baker. 1939 - Progress report of phosphate and other fertilizer investigations at the Aberdeen Branch Experiment Station, University of Idaho. Idaho Agr. Expt. Sta. Bul. 230 : 3.

WAKSMAN, Selman A. 1927 - Principles of soil microbiology. The Williams and Wilking Co., Baltimore.

WARINGTON, Katherine. 1923 - The effect of boric acid and borax on the broad bean and certain other plants. Ann. Bot $27: 630$.

WASHKO, John B. 1943 - Influence of borax and potash on alfalfa leaf yellows. Tenn. Agr. Expt. Sta. Circ. 83. 


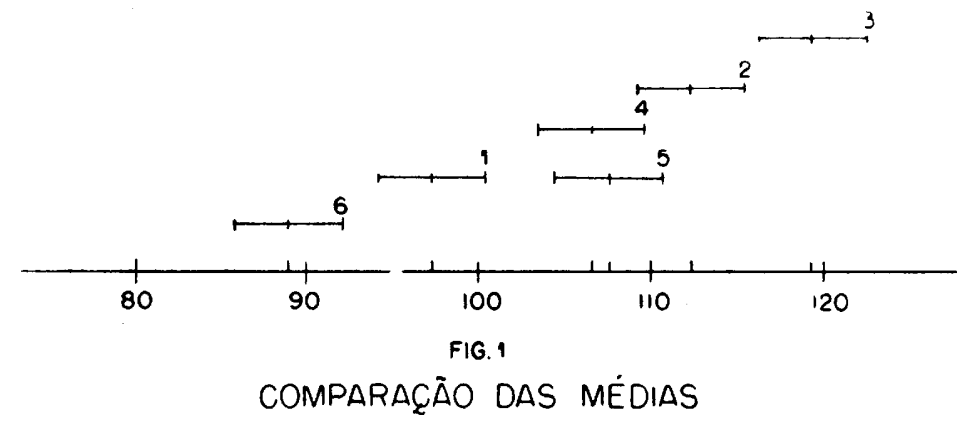




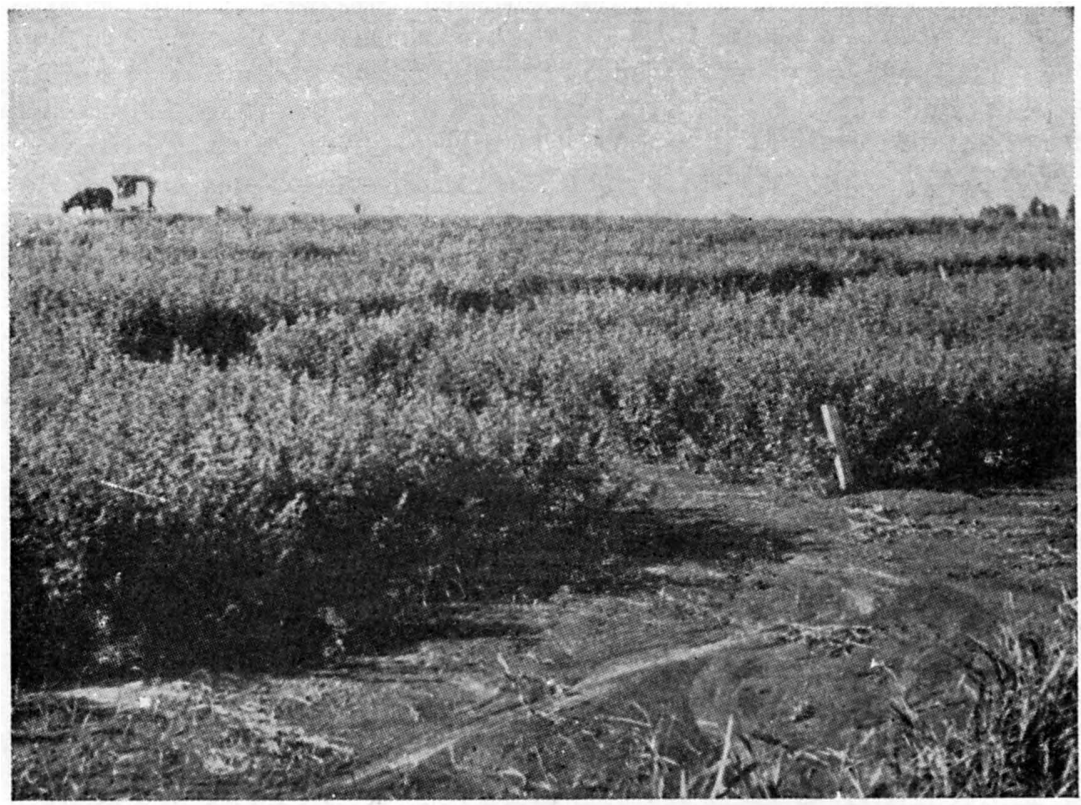

(Fig. 2) Foto de alfafa - Ensaio de campo

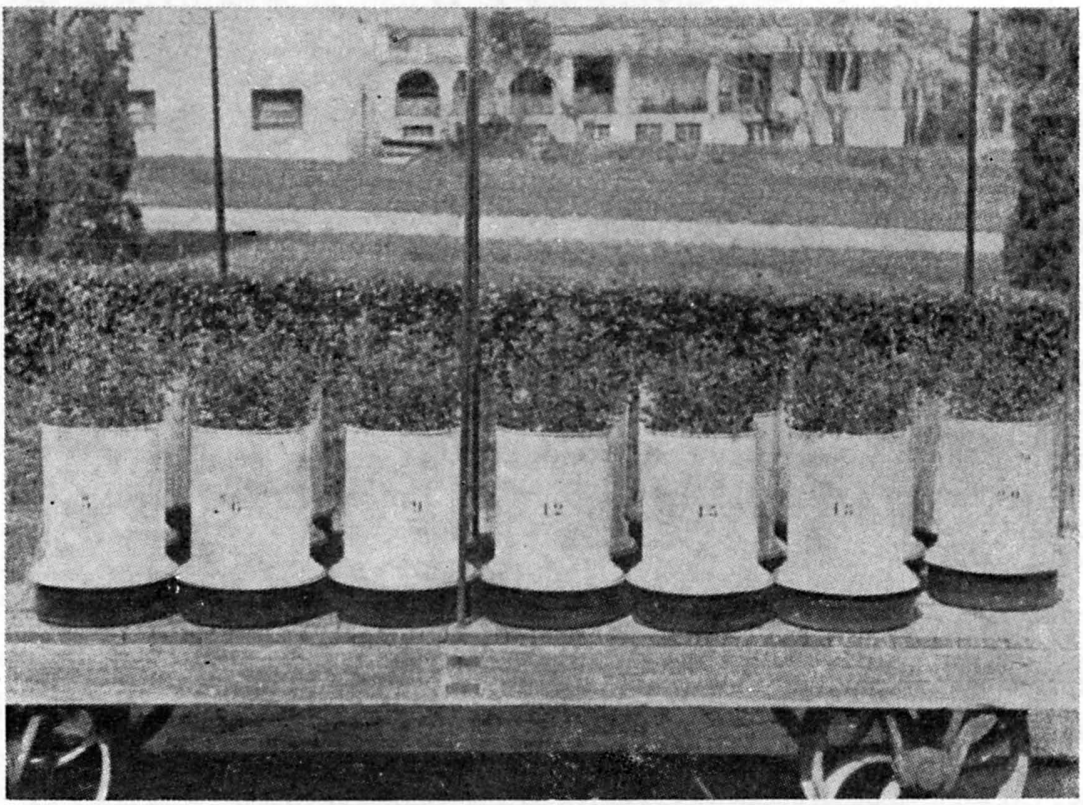

(Fig. 3) Foto de alfafa - Ensaio de vasos 


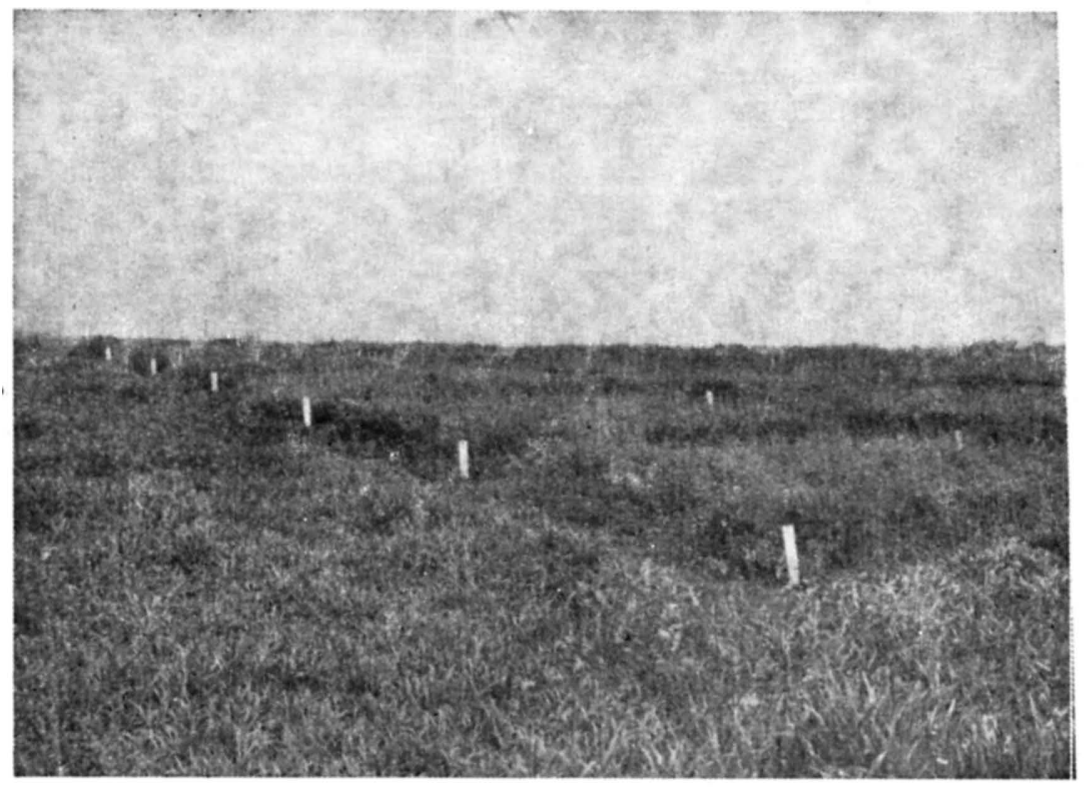

(Fig. 4) Foto de alfafa - Ensaio de campo

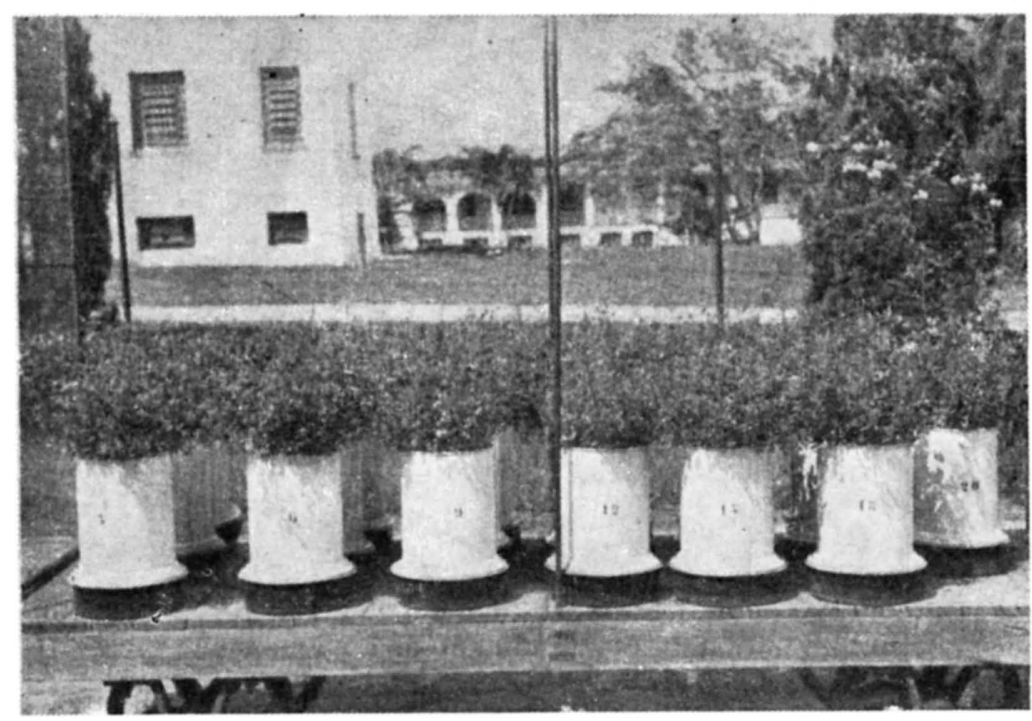

(Fig. 5) Foto de alfafa - Ensaio de vasos 
